\title{
Procesos de enseñanza de la lecto escritura en la educación básica primaria rural colombiana
}

\author{
Teaching processes of literacy of rural basic education teachers in the first grades of \\ schooling colombiana
}

\author{
Ferney de Jesús Morales Londoño \\ fercho125mora@hotmail.com \\ Código ORCID: 0000-0001-8861-6552 \\ Universidad Metropolitana Educación, Ciencia y \\ Tecnología (Umecit), Panamá
}

\author{
Sunny Raquel Perozo Chirinos \\ sunperozo@gmail.com \\ Código ORCID: 0000-0001-7574-1995 \\ Universidad del Zulia, Venezuela
}

Recibido: octubre 2020 / Arbitrado: noviembre 2020 / Publicado: enero 2021

RESUMEN

La presente investigación tuvo como propósito describir los procesos de enseñanza de la lectoescritura de los docentes de educación básica rural, en los primeros grados de escolaridad. En el diseño metodológico de la investigación, se retomó el holotipo de investigación descriptiva y el método de la holopráxis orientado desde la comprensión holística de la ciencia. Con un diseño de campo, transeccional contemporáneo. La población objeto de estudio fueron 50 docentes de aulas de las dos (2) instituciones educativas rurales, que llevan por nombre la I E R Pantanillo y I E R Zoila Duque Baena, ubicadas en el municipio de Abejorral, en el departamento de Antioquia. Para la recolección de los datos se utilizó la técnica de la observación directa no participante mediante una guía de observación. Los resultados indicaron que la mayoría de los docentes emplean los métodos sintéticos, lo que les mantiene anclados a metodologías tradicionales de repetición y memorización como procesos básicos para la adquisición del código.

Palabras clave:

Calidad educativa; lectoescritura; procesos de enseñanza; educación básica rural

ABSTRACT

The purpose of this research was to describe the teaching processes of literacy of rural basic education teachers in the first grades of schooling. In the methodological design of research, the holotype of descriptive research and the holopráxis method oriented from the holistic understanding of science was resumed. With a field design, contemporary transferal. The population under study was 50 classroom teachers from the two (2) rural educational institutions, named after the I E R Pantanillo and I E R Zoila Duque Baena, located in the municipality of Abejorral, in the department of Antioquia. For the collection of data, the technique of non-participating direct observation was used through an observation guide. The results indicated that most teachers use synthetic methods, which keeps them anchored to traditional repetition and memorization methodologies as basic processes for code acquisition. 


\section{INTRODUCCIÓN}

Hoy más que nunca la educación es un pilar fundamental en el desarrollo de la sociedad, de allí que sean diversos los temas que al respecto podrían tratarse: fines educativos, políticas educativas, cobertura, eficiencia, entre otros; sin embargo, uno de los que de forma particular ocupa un lugar predominante en la agenda educativa es la calidad, la cual involucra, dentro del sistema educativo un sinnúmero de factores determinantes para lograrlo.

En el contexto del aula y de manera más específica en la construcción de los aprendizajes de los estudiantes, la calidad educativa se encuentra asociada a factores tales como: la formación del maestro y la actitud y motivación que tenga ese profesional para asumir de manera positiva su actividad docente; de esos factores depende el tipo de enseñanza que se ofrece lo cual repercute en el progreso o desarrollo que alcance el país.

De esta manera se demuestra que la educación no es simplemente la trasmisión de información en un sitio específico, sino de un tipo de información que conforma procesos complejos que parten de la planificación y decisiones bien pensadas sobre la teoría, metodologías, estrategias y recursos empleados durante el proceso de enseñanza, así como del método que utilice el docente para realizar su labor.

Cuando se habla de enseñanza de la lectoescritura, esta importancia de la educación se hace aún más palpable, ya que es a través de ese proceso como, desde los primeros grados de formación, el niño comienza a ver el mundo desde otra óptica, a captar la realidad desde la visión de la indagación y la curiosidad por un nuevo panorama que abre sus puertas a los nuevos saberes, introduciéndole desde la lectura y la escritura, en otras realidades.

Sin embargo y pese a esa gran relevancia que tiene la enseñanza de la lectoescritura, se observa con preocupación como hoy día se sigue asumiendo la educación con métodos tradicionales donde predomina la repetición, la memorización de datos, fórmulas, información o reglas que a la larga se vuelven insuficientes para afrontar una realidad compleja que demanda del individuo mucho más que simple "capacidad para almacenar datos".

Es así como se plantea la necesidad de que la educación más allá de impartir contenidos en diferentes áreas, posibilite el desarrollo progresivo de habilidades del pensamiento y su estrecha vinculación entre éstas y la lectoescritura. Es decir, es desde el aula como se deben formar sujetos autónomos, críticos con capacidades suficientes para analizar, interpretar, sintetizar $\mathrm{y}$ argumentar con ideas y criterios propios y para lograrlo se debe contar con maestros bien preparados y actualizados.

Sin embargo, alcanzar ese propósito convierte a la calidad del proceso de lectoescritura en un tema relevante, presente por mucho tiempo en los planteamientos de organizaciones de corte mundial, como la Unesco (2011) y la Organización para la Cooperación y el Desarrollo Económico (OCDE 1990), para definir no sólo políticas educativas que cierren las brechas de desigualdad a través de programas de alfabetización, sino también para sensibilizar a los sistemas educativos en cuanto a que involucren la lectoescritura como eje transversal y 
articulador de los currículos escolares, por ser considerada como un factor determinante para la construcción de los saberes infantiles desde las diferentes áreas del conocimiento.

\section{Contexto}

Actualmente la educación es el motor para el desarrollo económico, social e integral de los pueblos (Espinosa y Vaca, 2012). Freire (sf c.p. Olivera y Orellana, 2005) va un poco más allá y plantea un concepto de educación centrado en "La praxis, la acción, la reflexión del hombre sobre el mundo para transformarlo" (p.37). Es así como la educación desde un concepto multidimensional no sólo es el medio para favorecer el cierre de brechas sociales, sino que es la vía por la cual las personas logran transformarse a sí mismos, a su entorno y a la sociedad.

De esta manera, la educación puede no sólo generar oportunidades de desarrollo y crecimiento de los pueblos, sino también la formación de ciudadanos conscientes de sus deberes y derechos, capaces de construir un nuevo tejido social donde predominen los valores esenciales de la sociedad, que puedan velar por la defensa de los derechos humanos, la estimación de la interculturalidad y el respeto por la diversidad, entre otros.

De acuerdo con Freire, (sf, cp Olivera y Orellana 2005) "Educar es hacer pensar, es transformación del sujeto y de las estructuras sociales en las cuales le tocará vivir" (s.n). Por ello, no toda educación recibida es buena y para que sea una educación de calidad debe estar revestida de pertinencia, valores, relevancia e integralidad. Cuando Freire se refiere a la educación como desarrollo del pensamiento, automáticamente está implícito el despliegue del proceso de lectoescritura, porque es a través de ese cúmulo de actividades como el individuo crece en lo interno, para desarrollar luego actividades creativas hacia su entorno.

En este sentido, hoy día hablar de educación de calidad plantea la necesidad de trabajar en el desarrollo de diferentes políticas educativas, que más allá de garantizar una educación para todos y con estándares de calidad en el marco de políticas economicistas, ayude a garantizar que todos los estudiantes aprendan a reconocerse como sujetos críticos y que las enseñanzas contribuyan a mejorar su calidad de vida en el lugar donde se desarrollan.

Al respecto la Unesco (2011) expone:

Recibir una educación de calidad a lo largo de toda la vida es un derecho congénito de cada niño, mujer u hombre. Por su parte, la educación, en particular la que se imparte a las niñas y las mujeres, contribuye a la consecución de todos los objetivos del desarrollo (p. 5).

De esta forma, garantizar el acceso a una educación de calidad, es una necesidad que apremia a todos los sistemas educativos en el mundo. Todas las personas sin excepción de ningún tipo, además de recibir una educación gratuita, tienen el derecho a recibir una educación de la mejor calidad.

De acuerdo con la Organización para la Cooperación y el Desarrollo Económico (OCDE 2004), una de las prioridades que tienen los sistemas educativos en el mundo y específicamente en América Latina es mejorar la calidad de la educación, como 
medio para avanzar en el desarrollo económico. Estas consideraciones implican que en los países latinos de forma permanente se trabaje en una articulación de las políticas educativas con las estrategias globales de desarrollo, y se propenda por la transformación permanente de los sistemas educativos para lograr una educación de mayor calidad y equidad.

Así mismo, la Organización de las Naciones Unidas para la Educación, la Ciencia y la Cultura (Unesco, 2011) dentro de sus objetivos señala que uno de los propósitos de la educación, es lograr construir un proyecto para América Latina y el Caribe, donde se dé la distribución igualitaria de la educación, concebida ésta como educación de paz, de respeto a la diversidad, como afirmación de la libertad y de la democracia. Propósito que está muy lejos de lograrse, ya que se continúa viendo la desigualdad socio-económica y las bajas cifras en materia educativa.

En Colombia, afortunadamente el tema de una educación de calidad, es uno de los puntos esenciales y vitales de la agenda política educativa, que debido a la importancia que se le da, ocupa un lugar privilegiado como uno de los grandes objetivos del Estado, convirtiéndose en numerosos esfuerzos en inversión y trabajo de comisiones de expertos que buscan mejorar el sistema educativo en la educación básica y secundaria.

En este sentido, el Ministerio de Educación Nacional de Colombia (c.p. Bautista, Gamboa y Sanabria ,2014) precisa la calidad como "Eficacia" y define la educación de calidad, como aquella que logra que los estudiantes aprendan lo que deben aprender; es decir, lo que está determinado en los estándares de competencias de las diferentes áreas, y esto debe evidenciarse a través de los resultados de las diferentes pruebas estandarizadas internas y externas que presentan los estudiantes en Colombia.

En otras palabras, desde una mirada técnico-instrumental, la evaluación de la calidad educativa en Colombia se ve reflejada en los resultados obtenidos por los estudiantes en las pruebas internas "Saber" y pruebas externas Programme for internacional Student Assessment (PISA). Al respecto, al hacer un análisis de los promedios obtenidos en los últimos 5 años en estas pruebas, se evidencia que, en áreas como matemáticas, lenguaje y ciencias naturales, se tienen avances significativos en las competencias que evalúan cada una de estas áreas, pero muy a pesar, estos resultados no son los esperados dentro de las metas de calidad del MEN.

De allí que estudiosos de la materia plantean la necesidad de mejoras y muy especialmente de la lectoescritura. Igualmente, se argumenta que podrían ser mejores los resultados en educación si en el área de lenguaje se fortalecieran el desarrollo de las competencias en lectura y escritura de los estudiantes, al considerar que estos procesos son fundamentales para la comprensión de conceptos y la aplicación de esas competencias en otras áreas del saber.

En este orden de ideas, preocupa que las competencias referidas a la lectoescritura sean unas de las más débiles en Colombia, no sólo por las implicaciones que esto trae en los resultados de pruebas a nivel nacional e internacional sino también por la transcendencia 
que tienen estas competencias en la formación humana, pues la "lectoescritura constituye las bases conceptuales determinantes para el desarrollo cultural del individuo" (Montealegre y Forero, 2006, p. 25) y son fundamentales para garantizar que los ciudadanos, puedan defender sus derechos participando abiertamente en una sociedad democrática.

Si bien en Colombia los contextos urbanos y rurales están marcados por grandes brechas de inequidad, donde los sectores rurales son los más afectados para garantizar a los estudiantes el acceso a la educación de calidad, en lo que respecta al contexto del municipio de Abejorral, las características geográficas, sociales, culturales, económicas y educativas son muy similares en ambos contextos al grado que dentro del Plan Educativo Municipal (PEM) (2017), se ha considerado todo el municipio como un territorio netamente rural, por tanto, no hay diferencias significativas entre estratos sociales, niveles educativos de los padres, tradiciones, costumbres; entre otros aspectos, que puedan marcar grandes divergencias.

Sin embargo, uno de los aspectos que sí contrapone la parte educativa entre estos dos sectores, rural / urbano, son los modelos educativos a partir de los cuales se enseña en básica primaria. La ruralidad es atendida por docentes que trabajan con la metodología multigrado, con el modelo educativo flexible de "Escuela Nueva", mientras que en la urbana la metodología es graduada. En la metodología multigrado el docente atiende varios grados a la vez generalmente de $0^{\circ}$ a $5^{\circ}$, mientras que en la escuela graduada el docente atiende un solo grado.

La metodología de "Escuela Nueva" en Colombia es un programa educativo dirigido, principalmente a las escuelas de las zonas rurales, caracterizadas por la alta dispersión de su población, producto de las condiciones socio-económicas propias de la zona, como son: dificultad para la movilización, niños que deben trabajar junto a los padres, condiciones geográficas que imposibilitan el acceso y otras que aunque no son objeto de esta investigación influyen también en el trabajo del maestro.

Por las características de cómo funciona la metodología de "Escuela Nueva", se resalta su importancia en la política de cobertura, pero también mucho se cuestiona la calidad de los procesos de enseñanza que allí se imparten, dado que la atención que tiene que dar el maestro de seis grados, dificulta los procesos de planeación y la didáctica de la enseñanza, realidad desde la cual se considera que la calidad educativa es una de las principales problemáticas del sistema educativo en la educación básica primaria de la ruralidad.

Por otra parte, los maestros del sector rural generalmente son profesionales recién egresados de formación complementaria de Escuelas Normales, mientras que los docentes del área urbana son profesionales de mayor experiencia, titulados en pregrado. Esa realidad ocasiona que el maestro no tenga la formación, el tiempo, ni la actitud requerida para asumir una actividad que involucre mayor compromiso y exija mayor preparación y dedicación en su aplicación, realización y evaluación.

La calificación de deficiente en el proceso de enseñanza de lectoescritura, es refrendada por diversos autores; por ejemplo Sawaya (2016), quien considera que la lectura y la escritura en la escuela se ha limitado e instrumentalizado en prácticas de 
decodificación, prescindiendo de otros usos, como "Procesos" que llevan a formas de construcción de sentidos, de interpretación y de la producción textual que se escapan a las formas escolares, al proyecto de sociedad y de individuo que se quiere inculcar.

Lerner (2001) también plantea que "La lectura aparece desgajada de los propósitos que le dan sentido en el uso social porque la construcción del sentido no es considerada como una condición necesaria para el aprendizaje" (p.120). Esta autora confronta las realidades de la enseñanza de la lectura hoy en día, donde la escuela sigue promoviendo prácticas de lectura anquilosadas en la sola decodificación y no logra despertar el interés ni la motivación de los estudiantes por leer y menos aún, por asumir una postura crítica que permita a través de lo que lee construir subjetividades.

En función de lo antes planteado surge la necesidad de dar respuesta a la siguiente pregunta de investigación: ¿Cómo son los procesos de enseñanza de la lectoescritura utilizados por los docentes de la educación básica rural del municipio de Abejorral Antioquia?

\section{Enseñanza y lectoescritura}

La enseñanza encierra un sinfín de elementos que vinculan al maestro $y$ al estudiante, en un solo propósito alrededor de la educación y es el aprendizaje. Este proceso no es vivido de igual manera en todos los escenarios escolares, algunos enfoques de enseñanza se centran en concepciones tradicionalistas, donde predomina la transmisión del conocimiento en simples repeticiones de conceptos, reglas o fórmulas y otros donde el conocimiento se construye desde el descubrimiento, el cual demanda un carácter crítico, cuestionador y autónomo del estudiante.

Desde esta perspectiva Berzosa (2004 c.p. Infante 2007) considera que "La enseñanza debe estar, orientada hacia el desarrollo de la capacidad creativa y el sentido crítico, tanto del estudiante como del maestro". (p. 33). Aquí los procesos de enseñanza se configuran basados en la construcción colectiva y en el intercambio permanente de saberes, expectativas e inquietudes de los maestros y los estudiantes y no sólo sobre la transmisión de volúmenes de información y datos.

De manera bastante similar, Sarmiento (2007) al relacionar la enseñanza con el desarrollo del pensamiento plantea que:

La educación no reside en el aprendizaje de muchos datos sino en capacitar la mente de manera que lo haga sobre aquello que no se encuentra en los libros (Sócrates Platón, Thomas s/f), la acción de enseñar no debe centrarse en el conocimiento sino en el desarrollo del pensamiento. (p. 49)

De igual forma, Zabalza (1990, c.p. Sarmiento 2007) alude a los procesos cognitivos que encierra la enseñanza y expresa que ésta también es comunicación, en la medida en que responde a un proceso estructurado, en el que se produce intercambio de información (Mensajes entre profesores y alumnos). En este sentido, desarrollo del pensamiento y comunicación son dos grandes propósitos que deben orientar los procesos de enseñanza de 
cualquier área, pero más aún en lectoescritura porque esa es la puerta para entrar al mundo de los diferentes saberes.

En una definición más cercana al aula y al propósito de la presente investigación, de acuerdo con Stenhouse (1991, c.p Sarmiento 2007), se entiende por enseñanza a las estrategias que adopta el maestro dentro de la escuela, para cumplir con su responsabilidad de planificar y organizar el aprendizaje de los niños, y aclara: "Enseñanza no equivale meramente a instrucción, sino a la promoción sistemática del aprendizaje mediante varios medios" ( $p$ 49). Mientras que la enseñanza de la lectura y la escritura, constituye una práctica social y cultural, porque son dos habilidades o destrezas a utilizar y aprender en unos contextos determinados (entre ellos los escolares), que condicionan la naturaleza de la propia educación y el tipo de relación que se establecen con las otras áreas del saber.

Por tener el presente trabajo el propósito de profundizar en el proceso de enseñanza de la lectoescritura en los primeros años de escolaridad (transición y primero), se toman para su realización los postulados realizados por Hernández, (2003), sobre los enfoques que debe tener la enseñanza en la educación inicial y en la básica primaria. El mencionado autor plantea que los propósitos de enseñanza de la educación inicial y de la básica, en su respectivo orden, deben contribuir en primer lugar al: "Desarrollo armonioso de las potencialidades del niño, tomando como base los intereses propios de esta edad" (p. 31) y en segundo lugar, promover "La integración efectiva de los niños a la sociedad" (p. 32).

La enseñanza de la lectoescritura, según palabras de Villamizar (1998), ha sido desde siempre, el momento inicial del sistema escolar porque es el primer peldaño de la escolaridad $y$, por tanto, una circunstancia particularmente definitiva en lo que se refiere a adecuación o inadecuación, éxito o fracaso en la escuela por parte del educando; por lo tanto, como todo primer momento de la formación académica, la lectoescritura resulta un indicador de mucha fuerza en cuanto a la posibilidad de partir de ella, para definir lo que será el futuro del nuevo escolar.

Dentro del nuevo marco teórico de la enseñanza de la lectura y la escritura, la Teoría de la Lingüística asume la lectura y la escritura como procesos. Esto implica que la lectoescritura es una actividad comunicativa continua del individuo en la sociedad y no simplemente un aprendizaje que se inicia en los primeros años (alfabetización), aunque la alfabetización es importante, abarca más que sólo aprender a leer y escribir, el objetivo es la adquisición de nuevos conocimientos, el desarrollo de saberes y el desempeño conforme se va encontrando con hechos de lectoescritura más complejos que requieran producir o interpretar textos orales o escritos que nunca había producido o interpretado antes.

En lo que respecta a la enseñanza de la lectoescritura, en los últimos años, los sistemas educativos a nivel mundial, han fijado su interés en el desarrollo de políticas de calidad educativas, centradas en el fortalecimiento de los procesos de enseñanza de la lectura y la escritura haciendo especial énfasis en los procesos de alfabetización. Sin embargo, la falta de unanimidad y de coherencia entre los gobiernos de diferentes países ha hecho que no se logren las metas esperadas.

Es preciso destacar otro aspecto vinculado con la formación de la criticidad. La Unesco 
(1993) plantea que los maestros deben enseñar a los estudiantes no sólo a reconocer palabras sino a construir sentido de lo que leen. Asimismo, los Lineamientos Curriculares del Área de Lenguaje en Colombia (1998), recalcan que el énfasis de la lectura y la escritura se debe poner en el desarrollo de competencias desde la comprensión lectora y la producción de textos.

La noción de competencia es
una categoría pensada desde la
constitución y formación de los
sujetos en diferentes dimensiones
de su desarrollo y se definen en
términos de las capacidades con
que un sujeto cuenta para
desempeñarse en una situación
específica.... (p.17)

Ahora bien, al hacer una desagregación del binomio lectoescritura, para determinar las particularidades que encierra la enseñanza de la lectura y la escritura se encuentra que de acuerdo con Josette (1993) aprender a leer, es desde el inicio, aprender a buscar el significado de un texto $(\mathrm{s} / \mathrm{n})$, ya no es sólo introducir letras o sílabas sin sentido para el lector. Es decir, no es un simple repetir o copiar sin un sentido, que responda a una necesidad comunicativa de ser entendido o establecer un intercambio con otros.

Desde otro punto de vista, Lerner (2001) plantea que "La lectura aparece desgajada de los propósitos que le dan sentido en el uso social porque la construcción del sentido no es considerada como una condición necesaria para el aprendizaje" (p.120). Las palabras de Lerner, orientan hacia la descontextualización que se hace de la lectura de la realidad e interés del educando; la lectura se convierte así en un simple objetivo a cubrir por el maestro.

Para aprender a leer se requiere que el maestro genere desde la enseñanza y la motivación del estudiante, la necesidad de hacerlo. De acuerdo con Rousseau (1712-1778, c.p. Soëtard 2011) "La lectura no es un fin en sí, sino que debe iniciarse en el momento oportuno -que puede ser muy diferente de un niño a otro- en el proceso de reapropiación intelectual del mundo" (s/n).

En otras palabras, la lectura más allá de la tradición lingüística y de algunas teorías psicológicas, donde se considera el acto de "Leer" como una decodificación del significado del texto, es considerada desde los Lineamientos Curriculares del MEN (1998) como:

Un proceso de interacción entre un sujeto portador de saberes culturales, intereses deseos, gustos, etcétera, y un texto como el soporte portador de un significado, de una perspectiva cultural, política, ideológica y estética particulares, y que postula un modelo de lector; elementos inscritos en un contexto: una situación de la comunicación en la que se juegan intereses, intencionalidades, el poder; en la que está presente la ideología y las valoraciones culturales de un grupo social determinado. (p. 27)

Algo similar sucede con la escritura, más que considerarse un acto de codificación de significados a través de reglas lingüísticas o de transcripción de conceptos, en los lineamientos del MEN (1998) se expone que: 
Se trata de un proceso que a la vez es social e individual en el que se configura un mundo y se ponen en juego saberes, competencias, intereses, y que a la vez está determinado por un contexto socio-cultural y pragmático que determina el acto de escribir: escribir es producir el mundo (p. 27)

Bajo la misma óptica, con relación a los procesos de escritura Hernández (2006 c.p. Benavides y Sierra 2013) considera que: "La escritura es un proceso cognitivo complejo que consiste en traducir ideas, pensamientos y/o afectos en discurso escrito coherente con fines comunicativos determinados" (p. 89). En consecuencia, la escritura más allá de ser un proceso en el que se adquiere un código, tiene sentido en tanto se conciba su enseñanza en la escuela desde la significación, lo cual conlleva al desarrollo de diversas habilidades del pensamiento.

Por ello, la enseñanza de la escritura, retomando a Hernández (2006) "Requiere desarrollarse atendiendo a diferentes elementos: los tópicos (¿Qué escribir?), lo retórico (¿Cómo escribirlo?) y lo comunicativopragmático (¿Para quién y para qué escribirlo?)" (p. 89). Desde esta perspectiva, la enseñanza de la escritura se enfoca en una escritura con sentido que tiene claro un propósito y una audiencia. Así la escritura permite al sujeto comprender el mundo y poder hacer representaciones del mismo.

En cuanto a lo pragmático, para Sawaya, (2016) "El aprendizaje de los usos sociales de la escritura se torna como una de las estrategias para la superación de la pobreza y del subdesarrollo" (p.10). Desde esta concepción, es necesaria una enseñanza de la escritura que permita la expresión y comunicación de ideas y pensamientos, que lleven a transformar las realidades y el mundo y no sólo a transcribirlo y reproducirlo. El punto de reflexión en este sentido, se centra en transformar el enfoque de enseñanza actual de la escritura en la escuela, el cual tiene gran predominancia en la forma y no en el uso.

De acuerdo con cada una de las diferentes posturas, en la presente investigación se asume un enfoque de escritura que trasciende el enfoque tradicional centrado en el manejo del código alfabético: fonema, sílaba y palabra, donde se ha puesto el énfasis en la relación sonido/grafía, por un enfoque en el que sobresale la significación, donde resulta necesario según el MEN (1998) "Generar espacios de significación en los que la escritura cobre sentido social, y el problema de la fonetización y el acceso al código alfabético serán una necesidad que aparece de manera natural" (p. 30).

En correspondencia con las posiciones de los diferentes autores, es necesario promover desde la educación inicial una enseñanza de la escritura centrada en la producción de textos, donde se exija movilizar más habilidades del pensamiento y se parta de situaciones de contexto como un producto comunicativo-social. Esta concepción de escritura resalta la importancia de que la enseñanza de la escritura en la escuela, permita ver la escritura como un acto de comunicación de ideas y de libre expresión.

Al respecto, Díaz Barriga y Hernández (2002) plantean que: 
Es necesario enseñar en contexto los procesos escriturales y los géneros retóricos, sin omitir aspectos comunicativos y funcionales, en vez de enfocarse en los microprocesos (gramática, estructura, ortografía, morfosintaxis, puntuación) en los productos $y$ en las evaluaciones superficiales (presentación, ortografía y gramática). (p. 89)

A nivel internacional, el planteamiento de Díaz Barriga y Hernández (2002) tiene gran incidencia en las nuevas políticas de calidad para la enseñanza de los procesos de lectura y escritura, donde se privilegia el uso de la lingüística textual con un enfoque semántico-comunicativo por encima del estructuralismo que da privilegios a la gramática y la sintaxis del lenguaje.

Se suma a lo anterior el planteamiento de Lerner (2001), quien señala que para que la lectura como objeto de enseñanza no se aparte demasiado de la práctica social que se quiere comunicar, es imprescindible "representar" o "re-presentar" en la escuela los diversos usos que ella tiene en la vida social (p. 1). Desde esta perspectiva en la presente investigación se asume un enfoque de lectura que trasciende el enfoque tradicional de lectura centrado en la sola decodificación. Esta concepción es también complementaria con la de Lerner (2001) quien plantea que "La lectura es indagar en la realidad para comprenderla mejor, es distanciarse del texto y asumir una postura crítica frente a lo que se dice y lo que se quiere decir, es sacar carta de ciudadanía en el mundo de la cultura escrita" (p. 115).

De igual forma, esta posición es coherente con lo expuesto en los Lineamientos
Curriculares en Colombia de 1998 donde el MEN, expone que:

Leer es un proceso de construcción de significados a partir de la interacción entre el texto, el contexto y el lector, en el cual el significado, a diferencia de lo que sostenía el modelo perceptivo motriz de la lectura, no está sólo en el texto, tampoco en el contexto ni en el lector, sino en la interacción de los tres factores, que son los que, juntos, determinan la comprensión ( $p$. 47).

Con relación a los currículos, en general la lectura y la escritura requieren estar plasmadas como un referente articulador o transversal de los planes de estudio, por lo que la enseñanza no debería realizarse de forma desarticulada desde todas las áreas del saber. Así mismo, las concepciones de lectura y escritura plasmadas en la fundamentación conceptual del plan de estudios de lengua castellana requieren estar enfocadas desde la significación y como prácticas sociales, sin estar limitadas al solo estructuralismo y la gramática tradicional.

Estas consideraciones de teóricos y del propio ente rector en materia educativa, han estado al margen de los procesos de enseñanza de la lectoescritura puesto que, en la educación actual, la preocupación del maestro se ha centrado más en definir qué métodos emplear para lograr la alfabetización de los alumnos, cuyo objetivo es fundamental para las instituciones escolares en la educación inicial, ya que así está dispuesto entre las labores del maestro dentro del aula. 
Así, la lectoescritura requiere ser abordada desde su dimensión comunicativa y comprensiva (Unesco 1993, p 2). Este planteamiento, aunque del 1993, hoy cobra gran vigencia en el currículo del área de lenguaje, toda vez que aún se privilegian los enfoques de enseñanza centrados en el estructuralismo: normativo/prescriptivo, cuando en realidad se requiere una enseñanza centrada en el fortalecimiento de la significación donde "Se haga evidente una serie de actuaciones o desempeños discursivos o comunicativos particulares asociados a las competencias lectoras y escritoras" (MEN, Lineamientos 1998, p. 28)

En la actualidad, desde los Lineamientos Curriculares (1998) se ha dispuesto de un enfoque de enseñanza, donde se espera que los docentes del área, realicen el proceso de enseñanza del lenguaje desde la significación de la lectoescritura y con una perspectiva pragmática, en donde "Se privilegien los modos de interactuar oralmente, de leer y de escribir de los estudiantes, fundamentos en la textolingüística" (MEN, 1998. p. 56). Así desde esta postura se concluye que se llega a las categorías gramaticales a través del análisis del uso; es decir de lo general a lo particular.

En este nuevo enfoque, la lectoescritura es planteada como un proceso analítico, interactivo, constructivo y estratégico, que demanda según Sawaya (2016), "La construcción de un lector y escritor competente donde la escuela se transforme en un espacio de lectura y escritura haciendo hincapié sobre sus usos sociales" (p. 14). Una posición totalmente opuesta a la enseñanza tradicional donde se privilegia lo memorístico (de normas y reglas ortográficas) y mecánico, sin darle mucha atención a la comprensión del texto o a la función comunicativa que debe cumplir la lectoescritura.

Adicional a ello, para obtener resultados más positivos en cuanto a la calidad educativa en el país, la enseñanza de la lectoescritura requiere dejar de ser vista sólo como una tarea exclusiva del docente de área de lenguaje, lo cual es corroborado por la Unesco (1993) cuando plantea que "La enseñanza de la lectoescritura está estrechamente vinculada con las demás asignaturas, donde los niños desde el principio interrogan los textos en su verdad de una manera crítica y creativa" (p. 3). Situación que acontece en el sistema educativo nacional, porque la lectoescritura es atendida única y exclusivamente por el docente de lengua y en las horas asignadas para ese fin.

\section{MÉTODO}

Esta investigación se considera de tipo descriptiva, con un diseño de campo, transeccional, contemporánea. La población estuvo conformada por 50 docentes de aulas de las dos (2) instituciones educativas rurales, que llevan por nombre la I E R Pantanillo y I E R Zoila Duque Baena, ubicadas en el municipio de Abejorral, en el departamento de Antioquia.

Para la recolección de los datos se utilizó la técnica de la observación directa no participante mediante una guía de observación.

En este sentido al no contar con una información previa y precisa que permitiera identificar cómo enseñan los maestros del sector rural a leer y a escribir en escuelas 
con metodología de "Escuela Nueva" del municipio de Abejorral, se desarrolló con los maestros un ciclo de observaciones de clase en diferentes momentos del año escolar, para identificar de acuerdo con sus prácticas de aula, los métodos más empleados en la enseñanza de la lectoescritura.

\section{RESULTADOS}

Con relación a los resultados del evento, a continuación se muestran los análisis realizados a la información y datos recopilados, para explicar los elementos relacionados con los procesos de enseñanza de la lectoescritura en educación inicial en las dos escuelas estudiadas, multigrados con metodología de "Escuela Nueva", ubicadas en el municipio de Abejorral del departamento de Antioquia.

En cuanto al proceso de facilitación de la enseñanza, el cual comprende un momento de inicio, de desarrollo y cierre, se pudo evidenciar lo siguiente:

En la Tabla 1 se muestra como del 100\% de maestros que fueron acompañados en el aula, el 58\% al inicio de la facilitación de los procesos de enseñanza de la lectoescritura en el primer grado de escolaridad, comienzan sus clases con el empleo de procedimientos propios del método sintético; donde prevalece el uso de la enseñanza del alfabeto, las sílabas y la pronunciación de las letras (fonético).

Igualmente, se observó que otro $17 \%$ de los maestros lo hacen con procedimientos propios de los métodos analíticos; es decir, de palabras y el global. Desde este método los maestros emplearon como actividades de entrada, la lectura de textos, la realización de preguntas de comprensión lectora y la escritura de palabras relacionadas con los textos $\mathrm{u}$ oraciones trabajadas en la clase, con una connotación pragmática y semántica.

Finalmente, el $25 \%$ restante de los docentes, comienza el proceso con procedimientos que no competen a la fase de inicio de los métodos sintéticos o analíticos, sino que pertenecen a estadios más avanzados de la clase, como lo es el desarrollo o cierre del proceso de facilitación; es decir, las actividades propuestas no se corresponden con el paso a paso planteado para el desarrollo de los métodos sintéticos, analíticos o mixtos, en su etapa inicial, ya que en los métodos sintéticos se parte del reconocimiento o identificación de una letra y en los analíticos y mixtos se comienza con el reconocimiento de una palabra, oración o un texto.

De esta forma, se pudo percibir que los maestros en su proceso de enseñanza de la lectoescritura, emplean mayoritariamente el uso de métodos tradicionales como el alfabético, fonético y silábico y dan predominancia a la enseñanza de las letras una a una, luego a la formación de sílabas y posteriormente las palabras y profundizan en su estructura gramatical o sintáctica. Sólo un porcentaje mínimo inicia el proceso de enseñanza de la lectoescritura con procedimientos desde métodos analíticos, partiendo de las palabras, las oraciones o textos, y dan mayor relevancia a las competencias pragmáticas y semánticas, centradas en la construcción del significado, antes que a las competencias sintácticas que se ocupa más de las formas y estructuras de las palabras. 
Tabla 1. Método de inicio en el proceso de enseñanza de la lectoescritura

\begin{tabular}{cccccc}
\hline & & Frecuencia & Porcentaje & $\begin{array}{c}\text { Porcentaje } \\
\text { válido }\end{array}$ & Porcentaje acumulado \\
\hline Válidos & No utiliza & 3 & 25,0 & 25,0 & 25,0 \\
& Método sintético & 7 & 58,3 & 58,3 & 83,3 \\
& Método analítico & 2 & 16,7 & 16,7 & 100,0 \\
& 12 & 100,0 & 100,0 & \\
\hline
\end{tabular}

Fuente: Elaboración propia (2020)

Con respecto al momento de desarrollo del proceso de enseñanza de la lectoescritura, el cual se refiere al período de tiempo donde se profundiza en actividades que permitan poner en práctica los conocimientos adquiridos en la etapa inicial, se observa en la Tabla 2 que el $75 \%$ de los maestros empleó procedimientos propios de los métodos sintéticos, lo que, en relación al porcentaje que tuvo el inicio del proceso de enseñanza, evidencia que el porcentaje aumenta en la cantidad de maestros que durante la etapa de desarrollo siguen el paso a paso de los métodos sintéticos.

El otro $17 \%$ de los maestros continúa con procedimientos propios de los métodos analíticos y finalmente, el $8 \%$ restante desarrolló esta parte del proceso mediante la combinación de procedimientos tanto de los métodos sintéticos como analíticos, ante lo cual se podría decir que estos docentes no tienen definidas las estrategias y actividades a realizar en la enseñanza de la lectoescritura.

En esta fase de facilitación del proceso de enseñanza es evidente, que los maestros en sus clases tienen mayor inclinación, por el desarrollo de actividades que se orientan según los procedimientos de los métodos sintéticos. Es decir, realizan actividades de lectura y escritura orientadas desde el principio alfabético, con predominio de la ejercitación y la memorización.

Los maestros que desarrollan la enseñanza desde métodos analíticos, avanzan en esta fase del proceso, a través de la decodificación de textos y profundización en el significado de las palabras. Es decir, van de lo particular a lo general.

Tabla 2. Método para el desarrollo del proceso de enseñanza de la lectoescritura

\begin{tabular}{llcccc}
\hline & Frecuencia & Porcentaje & $\begin{array}{c}\text { Porcentaje } \\
\text { válido }\end{array}$ & $\begin{array}{c}\text { Porcentaje } \\
\text { acumulado }\end{array}$ \\
\hline \multirow{2}{*}{ Válidos } & Método sintético & 9 & 75,0 & 75,0 & 75,0 \\
& Método analítico & 2 & 16,7 & 16,7 & 91,7 \\
& Método mixto & 1 & 8,3 & 8,3 & 100,0 \\
& Total & 12 & 100,0 & 100,0 & \\
\hline
\end{tabular}

Fuente: Elaboración propia (2020) 
De acuerdo con los resultados expuestos en la Tabla 3 se observa que en el cierre del proceso de enseñanza (de un objetivo relacionado con la lectoescritura en el primer grado de escolaridad), momento en el cual el docente busca que el estudiante pueda aplicar lo aprendido y demostrarlo en actividades de lectura y escritura espontanea, se evidencia que el $50 \%$ de los maestros emplean procedimientos propios del método sintético, lo que se detecta con relación al porcentaje de inicio y desarrollo del proceso de enseñanza, ya que el porcentaje disminuye en la cantidad de maestros que sigue el paso a paso del método sintético. El otro $33 \%$ de los maestros continúan el proceso mediante procedimientos propios del método analítico y finalmente el $17 \%$ restante no desarrolla esta parte del proceso, por lo que el paso a paso expuesto en los métodos queda sin culminar.

En este sentido, se puede inferir que los maestros en el proceso de enseñanza de la lectoescritura no son consistentes en el desarrollo de un método. Generalmente, se realizan diferentes procedimientos que se desprenden del principio alfabético donde se profundiza en los sonidos y formas de las letras y luego se trabajan diversos ejercicios para lograr la decodificación, la automatización y posteriormente la fluidez lectora. La repetición de estos pasos ausentes de los procesos de significación hace que los procesos de enseñanza de la lectoescritura sean rutinarios y de poca motivación para los niños que desean aprender a leer y escribir.

Tabla 3. Método para el cierre del proceso de enseñanza de la lectoescritura

\begin{tabular}{llcccc}
\hline & Frecuencia & Porcentaje & $\begin{array}{c}\text { Porcentaje } \\
\text { válido }\end{array}$ & $\begin{array}{c}\text { Porcentaje } \\
\text { acumulado }\end{array}$ \\
\hline Válidos & No utiliza & 2 & 16,7 & 16,7 & 16,7 \\
& Métodos sintéticos & 6 & 50,0 & 50,0 & 66,7 \\
& Métodos analíticos & 4 & 33,3 & 33,3 & 100,0 \\
& Total & 12 & 100,0 & 100,0 & \\
\hline
\end{tabular}

Fuente: Elaboración propia (2020)

Con relación a las actividades de enseñanza, los resultados expuestos en la Tabla 4 se muestra que el $83 \%$ de los maestros participantes de la investigación emplean actividades relacionadas con el método sintético para la enseñanza de la lectoescritura, el 9\% desarrollan actividades en el marco del método analítico y el $8 \%$ restante emplean actividades mixtas, retomadas de los métodos mencionados anteriormente.
En perspectiva, es evidente que las actividades empleadas por la mayoría de los maestros para el cumplimiento de un objetivo de enseñanza de lectoescritura se desarrollan desde los métodos sintéticos, donde se busca de principio a fin, trabajar en la ejercitación y memorización. Generalmente el proceso empieza con actividades basadas en la discriminación de sonidos y formas de las letras, luego la combinación de éstas para 
formar sílabas y después la combinación de éstas para formar palabras y oraciones.

Por su parte, los maestros que emplean el método analítico realizan actividades al inicio del proceso de enseñanza, dirigidas a promover la construcción de sentido y la significación en los procesos de lectura y escritura. Además, cumplen actividades de exploración de saberes previos, lo que busca desarrollar predicciones, inferencias, parafraseo, entre otras habilidades lectoras, esto marca la diferencia con relación a los maestros que emplean métodos sintéticos en el comienzo del proceso, donde se enfatiza directamente la enseñanza de las formas y sonidos de las letras.

En las actividades de desarrollo, en ambos métodos de enseñanza se trabajó fuertemente la escritura y la lectura de sílabas, palabras y oraciones, marcándose el desarrollo de la conciencia fonológica, la realización de tareas como la asociación de palabra- imagen, completar sílabas faltantes, encerrar letras y sílabas en un texto, entre otras.

En las de cierre, se evidenció tanto en los docentes que emplearon métodos sintéticos como analíticos que la falta de una buena planificación de la clase, no permite que las actividades de cierre evidencien cómo se culmina un objetivo de enseñanza, para la obtención de un producto o cómo se evalúa el proceso para poner de manifiesto los aprendizajes obtenidos.

Tabla 4. Actividades desarrolladas en el proceso de enseñanza de la lectoescritura

\begin{tabular}{llcccc}
\hline & Frecuencia & Porcentaje & $\begin{array}{c}\text { Porcentaje } \\
\text { válido }\end{array}$ & $\begin{array}{c}\text { Porcentaje } \\
\text { acumulado }\end{array}$ \\
\hline Válidos & Método sintético & 10 & 83,3 & 83,3 & 83,3 \\
& Método analítico & 1 & 8,3 & 8,3 & 91,7 \\
& Método mixto & 1 & 8,3 & 8,3 & 100,0 \\
& Total & 12 & 100,0 & 100,0 & \\
\hline
\end{tabular}

Fuente: Elaboración propia (2020)

Tabla 5. Materiales utilizados en el proceso de lectoescritura

\begin{tabular}{llccrc}
\hline & Frecuencia & Porcentaje & $\begin{array}{c}\text { Porcentaje } \\
\text { válido }\end{array}$ & $\begin{array}{c}\text { Porcentaje } \\
\text { acumulado }\end{array}$ \\
\hline Válidos & Método sintético & 4 & 33,3 & 33,3 & 33,3 \\
& Métodos analíticos & 4 & 33,3 & 33,3 & 66,7 \\
& Métodos mixtos & 4 & 33,3 & 33,3 & 100,0 \\
& Total & 12 & 100,0 & 100,0 & \\
\hline
\end{tabular}

Fuente: Elaboración propia (2020) 
En cuanto a los materiales empleados para la enseñanza de la lectoescritura, los resultados expuestos en la Tabla 5 indican que el $34 \%$ de los maestros acompañados en el aula, emplean materiales para la enseñanza de la lectoescritura relacionados con los métodos sintéticos, el otro 33\% utiliza materiales de los métodos analíticos y el 33\% restante de los maestros emplean materiales tanto de un método como de otro para desarrollar sus clases de lectoescritura.

\section{CONCLUSIONES}

Con el desarrollo de la presente investigación, se logra principalmente describir el proceso que desarrollan los maestros del sector rural para la enseñanza de la lectoescritura en el grado primero. De igual manera, a partir de la observación de las prácticas de enseñanza de los docentes, se identifican los procedimientos, las actividades y los materiales utilizados en ese proceso, lo que permitió concluir que son mayoría los docentes que emplean los métodos sintéticos, que les mantiene anclados a metodologías tradicionales que privilegian la repetición y memorización como procesos básicos para la adquisición del código desde un enfoque prescriptivo y normativo de la enseñanza de la lectoescritura.

En contraposición existe un menor número de maestros que emplean métodos analíticos, llevan a cabo el proceso promoviendo el desarrollo de habilidades de pensamiento como la observación, la comparación, la descripción y la narración, en otras palabras, ponen en juego el enfoque centrado en la significación y la construcción de sentido más allá de la apropiación del principio alfabético.
REFERENCIAS

Bautista, J., Gamboa, E. y Sanabria, C. (2014). Modelo de aseguramiento interno de la calidad para las instituciones de educación superior en el marco del mejoramiento continuo de la calidad de la educación superior en Colombia. MEN, Colombia

Benavides, D. y Sierra, G. (2013). Estrategias didácticas para fomentar la lectura crítica desde la perspectiva de la transversalidad REICE. Revista Iberoamericana sobre Calidad, Eficacia y Cambio en Educación, 11(3), 79-109

Díaz Barriga, F. y Hernández, G. (2002). Estrategias docentes para un aprendizaje significativo. Una interpretación constructivista. México DF: McGraw Hill Espinosa, O y Vaca, P. (2012). La educación como motor de desarrollo integral: la importancia del capital humano en el crecimiento económico y social de largo plazo. Econografos. Escuela de economía $\mathrm{N}^{\circ} 18$

Hernández, A. (2003). Introducción a las ciencias de la educación. 3a ed. Ediciones UAPA: Santo Domingo. Recuperado de https://vdocuments.site/angelhernandez-castillo-introduccion-a-lasciencias-de-la-educacion.html Infante, G. (2007). Enseñar Y Aprender: Un Proceso Fundamentalmente Dialógico De Transformación Revista Latinoamericana de Estudios Educativos (Colombia), 3(2)

Josette, J. (1993). Recomendaciones para mejorar comprensión lectora y de escritura de niños de áreas rurales y urbano marginales. Proyecto principal de educación en América Latina y el Caribe. Lectoescritura factor clave de calidad de la educación. Boletín 32 Santiago de Chile 
Lerner, D. (2001). Leer y escribir en la escuela: lo real, lo posible, lo necesario. México, Fondo de Cultura Económica

MEN. (1998). Lineamientos curriculares en lengua castellana. Colombia

Montealegre y Forero (2006). Desarrollo de la lectoescritura: adquisición y dominio 1. Universidad Nacional de Colombia y Universidad Externado de Colombia. Acta colombiana de psicología 9(1): 2540, 2006

OCDE (2004). Handbook for Internationally Comparative Education StatistIcs: Concepts, Standards, Definitions and Classifications. Paris, France. 2004 http://213.253.134.43/oecd/pdfs/browsei t/9604031E.PDF

OCDE. (1990). Schools and quality. An international report. Paris: OCDE

Olivera y Orellana (2005) Escuchemos a Paulo Freire: una mirada a la educación popular. Revista electrónica diálogos educativos. Año 5, No 10, 2005 ISSN 0718-1310

PEM. (2017) Plan educativo municipal de Abejorral. Periodo 2016 - 2019

Sarmiento, M. (2007). La enseñanza de las matemáticas y las tic. Una estrategia de formación permanente. Universitat Rovira i Virgili. ISBN: 978-84-690-8294-2 / d.l: t.1625-2007

Sawaya, S y Cuesta, C. (2016). Lectura y escritura como prácticas culturales: la investigación y sus contribuciones para la formación docente. Buenos Aires Argentina. Editorial de la Universidad de La plata

Soëtard, M. (2011). Jean-Jacques Rousseau. Luz y sombra espacio de arte. Recuperado de https://memiuxtec.wordpress.com 2011/01/07 > jean-jacques-rousseau

Unesco. (1993). Proyecto principal de educación en América Latina y el Caribe. Lectoescritura factor clave de calidad de la educación. Boletín 32 Santiago de Chile.

Unesco. (2011). La UNESCO y la Educación. "Toda persona tiene derecho a la educación"

Villamizar, G. (1998). La lectoescritura en el sistema escolar. Editorial Laboratorio Educativo. Cuadernos de Educación. Segunda edición. Caracas, Venezuela 